\title{
Critérios para avaliação de incapacidade laborativa na insuficiência cardíaca
}

\author{
Criteria for evaluation of incapacity to work in heart failure
}

\section{Rafael Augusto Tamasauskas Torres ${ }^{1}$, Raquel Barbosa Cintra ${ }^{2}$, Eduardo Costa Sá ${ }^{3}$}

Torres RAT, Cintra RB, Sá EC. Critérios para avaliação de incapacidade laborativa na insuficiência cardíaca / Criteria for evaluation of incapacity to work in heart failure. Rev Med (São Paulo). 2015 out.-dez.;94(4):282-8.

RESUMO: Introdução: A Previdência Social, no Brasil, é uma seguradora social que garante a renda do segurado quando ele perde sua capacidade laborativa. Dentre os benefícios concedidos temos a aposentadoria por invalidez, sendo que para sua concessão o segurado deve passar por perícia médica que comprove sua incapacidade total e permanente. Quatorze por cento das aposentadorias por invalidez concedidas nos últimos três anos ocorreram devido a doenças do aparelho circulatório, sendo que a via final das maiorias destas doenças é a insuficiência cardíaca. Objetivo: Identificar critérios para determinar incapacidade total e permanente em segurados portadores de insuficiência cardíaca congestiva em perícias previdenciárias. Metodologia: Revisão bibliográfica, sem limite de data, realizada de abril a agosto de 2014, utilizando os seguintes descritores: insuficiência cardíaca, seguro por invalidez e aposentadoria, nas seguintes bases de dados: Pubmed/Medline; BVS (Biblioteca Virtual de Saúde); Scopus; Web of Science; e Cochrane. A legislação previdenciária foi pesquisada. Resultados: Insuficiência cardíaca pode ser considerada incapacitante para mobilidade, tarefas complexas e tarefas de autocuidado, além de cursar com incapacidade cognitiva moderada ou severa. Insuficiência cardíaca como complicação pós-infarto é forte indicador para aposentadoria precoce, tendo como fator prognóstico positivo para retorno ao trabalho idade menor que 60 anos, fração de ejeção maior que $35 \%$, ausência de financiamento durante o afastamento, ausência de ataques de ansiedade e trabalho com baixa exigência física. Discussão: O Grau III ou IV da insuficiência cardíaca, segundo NYHA e a Previdência Social, cursa com incapacidade que, se permanente, dará direito à aposentadoria por invalidez. Exames complementares não devem ser usados de forma isolada. Idade maior que 60 anos, sexo feminino e alta carga de trabalho são fatores de mau prognóstico. Conclusão: Para a avaliação de capacidade laborativa na insuficiência cardíaca devem ser levados em consideração os achados clínicos, a fração de ejeção, a cognição do examinado, a exigência física do trabalho e as condições de retorno a este, a idade do periciando e a classificação funcional utilizada pela NYHA ou da Previdência Social.

Descritores: Insuficiência cardíaca; Seguro por invalidez; Aposentadoria.

\begin{abstract}
Introduction: Social Security in Brazil, is a social insurance that guarantees the income of the insured when he loses his job capacity. Among the benefits granted have a disability pension, and for being granted the insured it must undergo medical examination to prove their total and permanent disability. Fourteen percent of disability pensions granted in the last three years were due to diseases of the circulatory system, and the final pathway of the majority of these diseases is heart failure. Objective: To identify criteria for determining total and permanent disability in patients with congestive heart failure in social security skills. Methodology: Literature review, no limit date, held from April to August 2014, using the following key words: heart failure, disability and retirement, the following databases: Pubmed/ Medline; BVS (Virtual Health Library); Scopus; Web of Science; and Cochrane. Pension legislation was investigated. Results: Heart failure is crippling to mobility, complex tasks and self-care tasks, and present with moderate or severe cognitive impairment. Heart failure as a complication after infarction is a strong indicator for early retirement, taking as a positive prognostic factor for return to work age less than 60 years, a fraction ejection greater than $35 \%$, lack of funding during the removal, absence of anxiety attacks and work with low physical demands. Discussion: The Grade III or IV heart failure progresses with a disability which, if permanent, will right to disability retirement. Complementary exams should not be used in isolation, and greater than 60 years old, female and high workload are poor prognostic factors. Conclusion: The evaluation of work capacity in heart failure should be taken into consideration clinical, ejection fraction, the cognition, physical demands of the job and conditions of this return, the age and NYHA functional classification or Social Security classification used.
\end{abstract}

Keywords: Hearth failure; Insurance, disability; Retirement.

1. Residente do $2^{\circ}$ ano de Medicina do Trabalho do Hospital das Clínicas da Faculdade de Medicina da Universidade de São Paulo - HCFMUSP.

2.Professora Convidada do Curso de Especialização em Medicina Legal e Perícias Médicas do Departamento de Medicina Legal, Ética Médica, Medicina Social e do Trabalho da Faculdade de Medicina da Universidade de São Paulo.

3.Professor Convidado do Curso de Especialização em Medicina Legal e Perícias Médicas, Medicina do Trabalho e Medicina do Tráfego do Departamento de Medicina Legal, Ética Médica, Medicina Social e do Trabalho da Faculdade de Medicina da Universidade de São Paulo. Autor para correspondência: Rafael A. T. Torres. Rua Joaquim Oliveira Freitas, 1644 - A. Vila Mangalot. São Paulo, SP. CEP: $05133-004$. E-mail: rafaeltamatorres@yahoo.com.br 


\section{INTRODUÇÃO}

Previdência Social é uma seguradora social que garante a renda do segurado quando ele perde sua capacidade laborativa ${ }^{1}$. Dentre os benefícios concedidos pela Previdência Social temos as aposentadorias, as quais são pagamentos mensais e vitalícios efetuados ao segurado por motivo de tempo de contribuição, idade, invalidez permanente ou trabalho exercido sobre condições especiais que prejudiquem a saúde ou integridade física ${ }^{2}$. Quanto à invalidez, "tem direito à aposentadoria por invalidez $\mathrm{o}$ segurado que, estando ou não em gozo de auxílio-doença, é considerado incapaz para o trabalho e insuscetível de reabilitação para o exercício de atividade que lhe garanta a subsistência"2.

Para a concessão deste benefício (aposentadoria por invalidez) o segurado deve passar por uma perícia médica que comprove sua incapacidade ${ }^{3}$. Segundo o Conselho Regional de Medicina do Estado de São Paulo, a perícia médica é caracterizada como ato médico pois exige conhecimento técnico da Medicina, sendo uma atividade médico-legal que é responsável pela produção da prova técnica em procedimentos administrativos e/ou em processos judiciais ${ }^{4}$.

No caso da Previdência Social a concessão de aposentadoria por invalidez dependerá da verificação da condição de incapacidade, mediante exame médico-pericial a cargo da Previdência Social, podendo o segurado, às suas expensas, fazer-se acompanhar de médico de sua confiança ${ }^{5}$.

O Anuário Estatístico da Previdência Social ${ }^{6}$ mostra os seguintes dados a respeito das concessões de aposentadorias urbanas por invalidez (Tabela 1).

Tabela 1. Quantidade de aposentadorias urbanas totais por invalidez concedidas, segundo os capítulos da Classificação Internacional de Doenças (CID), três famílias de CID com maior frequência - 2011/2013, no Brasil

\begin{tabular}{|c|c|c|c|c|}
\hline & 2011 & 2012 & 2013 & $\begin{array}{c}\text { Total de benefícios } \\
(2010 \text { a 2013) }\end{array}$ \\
\hline Total de benefícios concedidos & 159.377 & 158.932 & 166.910 & 485.219 \\
\hline Doenças do sistema osteomuscular e do tecido conjuntivo & 23.485 & 23.839 & 23.286 & 70.610 \\
\hline Doenças do aparelho circulatório & 23.653 & 23.140 & 23.494 & 70.287 \\
\hline Transtornos mentais e comportamentais & 14.535 & 13.023 & 12.068 & 39.626 \\
\hline
\end{tabular}

Fonte: adaptada de Anuário Estatístico da Previdência Social, 2011 e $2013^{6}$.

Nesse contexto temos que as doenças do aparelho circulatório correspondem a $14 \%$ das aposentadorias urbanas concedidas nos três anos estudados, tendo, em número relativo, o mesmo nível de importância das doenças do sistema osteomuscular e do tecido conjuntivo, o que torna o estudo destas patologias importante, inclusive com critérios para avaliação pericial.

No Brasil, a fonte oficial de dados não possibilita a obtenção da porcentagem de casos de doenças cardíacas incluídas no universo das doenças do aparelho circulatório. Desta maneira a insuficiência cardíaca (IC) foi eleita neste estudo por fazer parte deste universo e representar situação frequentemente observada nas perícias e pela dificuldade de determinação da incapacidade laboral por falta de critérios claros.

A insuficiência cardíaca (IC) é a via final comum da maioria das doenças que acomete o coração ${ }^{7}$, tendo inúmeras etiologias como doença isquêmica, hipertensão arterial, doença de Chagas, cardiomiopatia, uso de drogas, toxinas, doenças endócrinas, doenças nutricionais, infiltrativas e outras doenças (fístulas artério-venosas, anemias, doença de Paget, etc. $)^{8}$. Em 2007 a IC foi a terceira causa de internação no Brasil pelo Sistema Único de Saúde (SUS), sendo a causa mais frequente de internação por doenças cardiovasculares ${ }^{7}$.

No Brasil a principal etiologia da IC é a cardiopatia isquêmica crônica associada à hipertensão arterial, sendo que em algumas localidades ainda existem formas de IC associadas à Doença de Chagas, endomiocardiofibrose e a cardiopatia valvular reumática, que são situações especiais de IC em nosso meio ${ }^{7}$.

Para IC, a classificação mais conhecida é a da New York Heart Association (NYHA), apresentada no Quadro $1^{9}$. 
Torres RAT, et al. Critérios para a avaliação de incapacidade laborativa na insuficiência cardíaca.

Quadro 1- Classes funcionais da NYHA

\begin{tabular}{ll} 
Classe funcional & Sintomas \\
\hline Classe I & Paciente com insuficiência cardíaca mas sem limitação durante as atividades habituais \\
Classe II & Pacientes com leve limitação às atividades habituais \\
Classe III & Pacientes com grande limitação funcional durante as atividades habituais, mas sem sintomas em repouso \\
Classe IV & Pacientes com sintomas em repouso \\
\hline
\end{tabular}

Fonte: adaptada de Oliveira e Porto, $2005^{9}$

Embora haja uma progressão com o passar do tempo, a classe funcional pode variar durante a evolução. Pacientes em classe III ou IV podem passar para classe I ou II dependendo do tratamento empregado, sendo o motivo da grande utilização desta classificação nos ensaios ${ }^{9}$.

A evolução da doença e a sintomatologia dependem de vários dados clínicos e laboratoriais. Barreto e Ramires ${ }^{10}$ afirmam que têm melhor evolução aqueles com maior capacidade física, e que dados de função cardíaca também identificam pacientes de pior prognóstico, como menor débito cardíaco, maior pressão capilar pulmonar, maior resistência periférica, menor fração de ejeção, sendo que tais fatores aumentam a mortalidade. Dados sobre anatomia também são úteis nesta estratificação, pois cardiomegalia, diâmetros ventriculares e atriais maiores identificam grupos de maior risco.

No entanto, analisando apenas uma variável da doença não é possível predizer a capacidade para determinada atividade, como demonstrado pelos estudos The SOLVD Investigator ${ }^{11}$ e Pfeffer et al. ${ }^{12}$, que após avaliarem pacientes assintomáticos com fração de ejeção menor que $40 \%$ concluíram que nem todos os portadores de disfunção ventricular possuem limitação da atividade física.

O Manual Oficial em Saúde do Servidor Público Federal classifica como cardiopatia grave, para concessão de alguns benefícios específicos, o servidor com classe funcional III ou IV da NYHA e/ou com fração de ejeção ao ecocardiograma menor que $40 \%{ }^{13}$.

Percebe-se então que, apesar das doenças circulatórias representarem $14 \%$ de deferimento de aposentadorias por invalidez no Brasil, faltam estudos sobre a associação destas doenças, em particular a insuficiência cardíaca, com a avaliação de incapacidade para concessão de aposentadorias.

\section{OBJETIVO}

Identificar critérios para determinar incapacidade total e permanente em segurados portadores de insuficiência cardíaca congestiva em perícias previdenciárias.

\section{MÉTODO}

No presente estudo foi realizada uma revisão bibliográfica, incluindo artigos publicados sem data definida, sendo o levantamento destes realizados no período de abril a agosto de 2014, utilizando os seguintes descritores encontrados no portal Descritores em Ciências da Saúde (DeCS):

$>$ Insuficiência cardíaca / heart failure;

$>$ Seguro por invalidez / disability;

> Aposentadoria/retirement.

As bases de dados consultadas foram:

> PUBMED/MEDLINE;

$>$ BVS (Biblioteca Virtual de Saúde);

$>$ SCOPUS;

$>$ WEB OF SCIENCE;

$>$ COCHRANE.

Além disso, foi também pesquisada a legislação previdenciária em vigor.

Foram excluídos do presente estudo os artigos que não estavam disponíveis na língua inglesa ou portuguesa e artigos que não eram disponíveis de forma gratuita nas bases de dados pesquisadas.

\section{RESULTADOS}

Ao utilizar os descritores foram encontrados 9 artigos no Pubmed, 0 artigo na BVS, 13 artigos na Scopus, 8 na Web of Science e 0 na Cochrane. Ao selecionar os que versavam sobre o tema do estudo, foram selecionados respectivamente 3, 0, 5, 4 e 0 artigos. Ao excluir os artigos repetidos, 7 artigos foram selecionados, listados no Quadro 2.

Os critérios para incapacidade contida na legislação da Previdência Social foram pesquisados, sendo que a insuficiência cardíaca consta apenas na Orientação Interna $\mathrm{n}^{\mathrm{o}}$ 081, de 15 de Janeiro de $2003^{14}$, listada no Quadro 2. 
Torres RAT, et al. Critérios para a avaliação de incapacidade laborativa na insuficiência cardíaca.

Quadro 2- Artigos e legislação selecionados pelo método

\begin{tabular}{ll}
\hline Autor (ano) & Título \\
\hline Dimond (1962) & Assessment of functional recovery of men surviving first myocardial infarction \\
Nielsen et al. (2004) & $\begin{array}{l}\text { A prospective study found impaired left ventricular function predicted job retirement after } \\
\text { acute myocardial infarction }\end{array}$ \\
Slebus et al. (2007) & Prognostic factors for work ability in sicklisted employees with chronic diseases \\
Serqueira (2009) & Aposentações por doença cardíaca em Portugal (2002-2008) \\
Rywik et al. (2011) & Characteristics of the heart failure population in Poland \\
Hung et al. (2012) & $\begin{array}{l}\text { Association of Chronic Diseases and Impairments with Disability in Older Adults: A Decade of } \\
\text { Change? }\end{array}$ \\
Gure et al. $(2012)^{21}$ & The Prevalence of Cognitive Impairment in Older Adults with Heart Failure \\
Brasil. Ministério da & Orientação Interna no 081, de 15 de Janeiro de 2003
\end{tabular}

Dimond $^{15}$ analisou o retorno ao trabalho de 348 pacientes em Detroit (sem avaliar ocupação) após um infarto agudo do miocárdio. A coorte foi realizada com pacientes com documentação para infarto entre 1940 e 1957, sendo os mesmos seguidos por 10 anos. Após o episódio, 74 pacientes aposentaram por invalidez, sendo que 10 destes foram devido a insuficiência cardíaca congestiva e 33 devido a quadro anginoso. Concluiu que, se o paciente apresenta uma boa recuperação do primeiro infarto, a maioria apresenta muitos anos livre de sintomas, tendo uma vida produtiva adequada. Mesmo com hipertensão, alterações eletrocardiográficas ou angina aos esforços, em condições apropriadas de trabalho, os pacientes devem retornar para este de maneira precoce. Relata que o aumento cardíaco atribuído a insuficiência cardíaca como complicação pós-infarto ou hipertensão, angina severa e persistente e aneurisma de ventrículo são fortes indicadores para aposentadoria precoce.

Nielsen et al. ${ }^{16}$ estudaram pacientes sobreviventes de infarto do Departamento de Cardiologia do Hospital Universitário de Herlev (Dinamarca) entre 1990 e 1993 que estavam empregados ou trabalhavam algum período deste estudo. Concluíram que a diminuição da fração de ejeção (menor que 35\%) após infarto é um determinante do prognóstico de aposentadoria. Concluiu também que ser do sexo feminino e ter uma carga alta de trabalho é um fator de vulnerabilidade para diminuição da fração de ejeção.

Slebus et al. ${ }^{17}$ em artigo de revisão, levantando artigos de 1990 a 2006, ao estudar infarto agudo do miocárdio e retorno ao trabalho encontraram como fator prognóstico positivo para retorno ao trabalho idade menor que 60 anos, fração de ejeção maior que $35 \%$, ausência de financiamento durante o afastamento, ausência de ataques de ansiedade e trabalho com baixa exigência física.

Serqueira ${ }^{18}$ avaliou os benefícios recebidos pelos funcionários do Estado em Portugal, entre eles as aposentadorias por invalidez concedidas entre 2002 e 2008. Elas são decididas em juntas médicas com dois ou três médicos. O autor encontrou incapacidade para o trabalho devido a insuficiência cardíaca em cerca de 4,50 a 7,50\% dos funcionários ativos doentes durante estes anos (valor mínimo de 4,47 \% em 2008 e valor máximo de 7,40\% em 2003, com média de 3.000 aposentadorias por ano) com uma elevada diferença entre os sexos, sendo que os homens variaram entre 7,00 e $12,00 \%$ do seu total enquanto as mulheres variaram entre 2,77 e 5,93\%. Foram aposentados $23,08 \%$ dos doentes (360) por insuficiência cardíaca sendo 203 mulheres (56,39\% do grupo) e 157 homens (43,61\%).

Rywik et al. ${ }^{19}$ em estudo multicêntrico na Polônia avaliaram 822 pacientes com insuficiência cardíaca, com média de 68 anos, observando que um quarto desta população foi aposentada por invalidez, sendo que $90 \%$ destas aposentadorias foram por insuficiência cardíaca. Concluiu que $32 \%$ da população tinha insuficiência cardíaca devido doença isquêmica e 38\% (70\% cumulativo) devido doença isquêmica associada com hipertensão.

Hung et al. ${ }^{20}$, ao analisar um braço do estudo Health and Retirement Study (HRS) nos Estados Unidos, analisaram adultos (12.652) que entraram na coorte em 
Torres RAT, et al. Critérios para a avaliação de incapacidade laborativa na insuficiência cardíaca.

1992 com 61 anos, sendo avaliados para este estudo com mais de 65 anos em 1998, 2004 e 2008. Encontrou associação entre insuficiência cardíaca e incapacidade para mobilidade, tarefas complexas e tarefas de autocuidado em todas as avaliações (em 1998 OR de 2,62; 3,03 e 2,60 respectivamente).

Gure et al. ${ }^{21}$ também analisaram um braço do estudo HRS usando dados de 2004, com pacientes de 51 anos ou mais $(n=9.663)$, obtendo OR de 1,52 (1,14-2,02;
IC de 95\%) em análise multivariada para incapacidade cognitiva moderada ou severa em presença de insuficiência cardíaca.

A Orientação Interna $n^{\circ} 081$, da Previdência Social, de 15 de Janeiro de $2003^{14}$ para médicos peritos previdenciários, na seção Cardiopatia Grave, coloca insuficiência cardíaca como uma das síndromes incapacitantes, sendo que para esta síndrome o periciando deve ser dividido em 4 graus de funcionalidade (Quadro 3):

Quadro 3- Graus de Funcionalidade segundo Orientação Interna n 81 da Previdência Social (2003)

\begin{tabular}{ll} 
Grau & Funcionalidade \\
\hline Grau I & Pacientes portadores de doença cardíaca sem limitação para a atividade física \\
Grau II & Leve limitação para a atividade física \\
Grau III & Dítida limitação para a atividade física \\
Grau IV & Doença cardíaca que os impossibilitam de qualquer atividade física
\end{tabular}

Fonte: adaptada de Orientação Interna $n^{\circ} 81$ de $2003^{14}$.

São considerados cardiopatas grave os pacientes em estágio III ou IV, ou que estiverem em estágio I ou II com doença em evolução após esgotada as possibilidades terapêuticas. Sobre os exames complementares consta que os achados fortuitos em exames complementares especializados não são por si só, suficientes para o enquadramento legal de Cardiopatia Grave, se não estiverem vinculados aos elementos clínicos e laboratoriais que caracterizem uma doença cardíaca incapacitante. Entretanto fração de ejeção menor que $35 \%$, ao ecocardiograma, já pode enquadrar a pessoa como cardiopata grave.

\section{DISCUSSÃO}

Existe pouca literatura específica sobre incapacidade laborativa em insuficiência cardíaca bem como a legislação é escassa. É inegável o impacto desta doença sobre as aposentadorias por invalidez e seu custo direto e, no caso deste estudo, indireto, refletido como gastos previdenciários, dias perdidos de trabalho ou mesmo perda da capacidade permanente para o trabalho, tanto no funcionalismo público (perícias administrativas) quanto no privado (segurados pelo Instituto Nacional da Previdência Social, perícias previdenciárias). Esses pontos foram levantados tanto por Serqueira ${ }^{17}$ ao retratar o quadro na esfera estatutária em Portugal, sendo possível traçar um paralelo com os gastos previdenciários por doença cardiovascular nos últimos 3 anos $^{14}$.

A legislação previdenciária brasileira carece de um protocolo para normatizar estas perícias. Está em fase de construção pela previdência uma cartilha para orientar os médicos peritos na avaliação de periciandos com insuficiência cardíaca. Atualmente há menção à insuficiência cardíaca grave na Orientação Interna $n^{\circ} 081$, de 15 de Janeiro de $2003^{14}$. Caso a insuficiência cardíaca seja enquadrada como cardiopatia grave, o segurado tem isenção de carência, mas não entra no âmbito de critérios bem estabelecidos e definidos de incapacidade.

A classificação utilizada pela previdência que divide a cardiopatia grave em graus ${ }^{14}$ assemelha-se muito à classificação utilizada pela NYHA, que já é consagrada na literatura médica científica ${ }^{9}$. No entanto, enquanto a segunda fala especificamente de insuficiência cardíaca, a primeira extrapola para todas as cardiopatias.

Esta mesma orientação ${ }^{14}$ alerta para a não utilização de exames isolados para definir por capacidade ou incapacidade, ao mesmo tempo que cita que fração de ejeção menor que $35 \%$ pode ser considerado como critério isolado para incapacidade em alguns casos, que é o mesmo valor exposto por Nielsen et al. ${ }^{16}$, que diz que fração de ejeção menor do que $35 \%$ é determinante no prognóstico de aposentadoria, acrescentando ainda que ser do sexo feminino e ter uma carga alta de trabalho é um fator de vulnerabilidade para diminuição da fração de ejeção, mas é um valor diferente do Manual de Perícia Oficial em Saúde do Servidor Público Federal (Brasil, 2010) que utiliza fração de ejeção menor que $40 \%$.

Slebus et al. ${ }^{17}$ ao estudar infarto agudo do miocárdio e retorno ao trabalho encontraram idade menor que 60 anos, fração de ejeção maior que $35 \%$, ausência de financiamento 
Torres RAT, et al. Critérios para a avaliação de incapacidade laborativa na insuficiência cardíaca.

durante o afastamento, ausência de ataques de ansiedade e trabalho com baixa exigência física como fatores de bom prognóstico para retorno ao trabalho. Visto que uma das principais etiologias da insuficiência cardíaca é isquemia miocárdica e hipertensão ${ }^{7,8,15,19}$, extrapolar os dados encontrados por Slebus et al. ${ }^{17}$ para insuficiência cardíaca seria possível e plausível, o que, aliás, complementa o discutido por Nielsen et al. ${ }^{16}$, Dimond ${ }^{15}$ e pela Previdência Social $^{14}$. Vale salientar no entanto, que todos os estudos mencionados acima alertam para a necessidade de avaliação da funcionalidade do periciando, sendo os exames usados como complementação ao raciocínio clínico, sem substituir o mesmo.

Um dado que chama atenção no estudo de Slebus et al. ${ }^{17}$ é a ausência de financiamento para o afastamento como fator de bom prognóstico, o qual levanta em sua própria discussão que, nos casos em que o paciente afastado não recebe auxílio financeiro, seu empenho na recuperação é maior, sendo um fator de melhor prognóstico. Dimond ${ }^{15}$ menciona que o trabalhador após infarto agudo do miocárdio (aqui extrapolado para insuficiência cardíaca) deve retornar assim que possível e precocemente ao trabalho, obviamente, em condições adequadas à sua nova condição.

Hung et al. ${ }^{20}$ encontraram incapacidade para mobilidade, tarefas complexas e de autocuidado associado à insuficiência cardíaca e Gure et al. ${ }^{21}$ encontraram insuficiência cardíaca como fator isolado para incapacidade cognitiva. Estes dois estudos são braços de uma mesma corte (HRS) mas seus resultados vão de encontro com incapacidades já bem descritas na literatura devido à própria fisiopatologia da doença ${ }^{7,9}$, como mostra o Quadro 4.

\section{REFERÊNCIAS}

1. Brasil. Ministério da Previdência Social. O que é a Previdência Social? [citado 19 abr. 2014]. Disponível em: http://www. mpas.gov.br/conteudoDinamico.php?id=59.

2. Brasil. Ministério da Previdência Social. Anuário Estatístico da Previdência Social. Brasília (DF): Empresa de Tecnologia e Informações da Previdência Social; 2011. p.13, 69-71.

3. Brasil. Ministério da Previdência Social. Lei No 8.213 de 24 de julho de 1991. Dispõe sobre os Planos de Benefícios da Previdência Social e dá outras providências. Diário Oficial da União, Brasília (DF), 25 jul. 1991. p.14809.

4. CREMESP. Resolução CREMESP N ${ }^{\circ} 126,17$ de outubro de 2005. Dispõe sobre a realização de perícia médica e dá outras providências. Disponível em: http://www.cremesp.org.br/?si teAcao $=$ LegislacaoBusca\&nota $=284$.

5. Brasil. Ministério da Previdência Social. Instrução Normativa
Quadro 4 - Resumo dos critérios de incapacidade e fatores de mau prognósticos encontrados para Insuficiência Cardíaca

Critérios determinantes de incapacidade

Classificação funcional da NYHA ou da Previdência Social grau III ou IV

Fração de ejeção menor que $35 \%$

Incapacidade cognitiva

Critérios de mau prognóstico

Alta carga de trabalho

Idade maior que 60 anos

Sexo feminine

\section{CONCLUSÃo}

A insuficiência cardíaca é uma síndrome que pode cursar com diversos graus e estágios de incapacidade (Grau I, II, III e IV; para mobilidade, tarefas complexas, tarefas de autocuidado e cognitiva). Exames complementares não devem ser usados de forma isolada para avaliar a capacidade destes periciandos, sendo que nesta avaliação devem ser levados em consideração os achados clínicos, a fração de ejeção (maior ou menor que 35\%), a cognição do examinado, a exigência física do trabalho e as condições de retorno a este, a idade do periciando e a classificação funcional utilizada pela NYHA ou da Previdência Social, sendo os critérios determinantes de incapacidade o grau III ou IV desta classificação, fração de ejeção menor que 35\% e incapacidade cognitiva.

INSS/PRES No 45 de 06 de agosto de 2010. Dispõe sobre a administração de informações dos segurados, o reconhecimento, a manutenção e a revisão de direitos dos beneficiários da Previdência Social e disciplina o processo administrativo previdenciário no âmbito do Instituto Nacional do Seguro Social - INSS. Diário Oficial da União, Brasília (DF), 6 ago. 2010.

6. Brasil. Ministério da Previdência Social. Anuário Estatístico da Previdência Social. Brasília (DF): Empresa de Tecnologia e Informações da Previdência Social; 2013. p.13, 69-71.

7. Bocchi EA, Marcondes-Braga FG, Ayub-Ferreira SM, Rohde LE, Oliveira WA, Almeida DR, et al. III Diretriz Brasileira de Insuficiência Cardíaca Crônica. Arq Bras Cardiol. 2009;93(1 supl.1):1-71.

8. Stewart AL, Greenfield S, Hays RD, Wells K, Rogers WH, Berry SD, McGlynn EA, Ware JE Jr. Functional status and 
Torres RAT, et al. Critérios para a avaliação de incapacidade laborativa na insuficiência cardíaca.

well-being of patients with chronic conditions: results from the medical outcomes study. J Am Med Assoc. 1989;262:90712.

9. Oliveira JG, Porto CC. Insuficiência cardíaca congestiva. In: Porto CP. Doenças do coração - prevenção e tratamento. 2a ed. Rio de Janeiro: Guanabara Koogan; 2005. Cap.56.

10. Barreto ACP e Ramires JAF. Insuficiência cardíaca. Arq Bras Cardiol. 1998;71(4):635-42.

11. The SOLVD Investigators. Effect of enalapril on mortality and the development of heart failure in asymptomatic patients with reduced left ventricular ejection fraction. N Engl J Med. 1992;327:685-91.

12. Pfeffer MA, Braunwald E, Moye LA, Basta L, Brown EJ Jr, Cuddy TE, Davis BR, Geltman EM, Goldman S, Flaker GC, Klein M, Lamas GA, Packer M, Rouleau J, Rouleau JL, Rutherford J, Wertheimer JH, Hawkins M. Effect of captopril on mortality and morbidity in patients with left ventricular dysfunction after myocardial infarction. Results on the survival and ventrivular enlargement trial (SAVE). N Engl J Med. 1992;327:669-77.

13. Brasil. Ministério do Planejamento, Orçamento e Gestão. Capítulo VII: doenças especificadas em leis. Manual de Perícia Oficial em Saúde do Servidor Público Federal, 2010. Diário Oficial da União, Brasília (DF), 23 mar. 2010.

14. Brasil. Ministério da Previdência Social. Orientação interna INSS/PRES N $\mathrm{N}^{\circ} 81$ de 15 de janeiro de 2003. Manual de avaliação das doenças e afecções que excluem a exigência de carência para a concessão de Auxílio-doença ou aposentadoria por invalidez.

15. Dimond GE. Assessment of functional recovery of men surviving first myocardial infarction. Fundamentals of clinical cardiology. 1962;65(6):832-8.

16. Nielsen FE, Sorensen HT, Skagen K. A prospective study found impaired left ventricular function predicted job retirement after acute myocardial infarction. J Clin Epidemiol. 2004;57:837-42.

17. Slebus FG, Kuijer PPFM, Willems JHBM, Sluiter JK, Frings-Dresen MHW. Prognostic factors for work ability in sicklisted employees with chronic diseases. Occup Environ Med. 2007;64:814-9.

18. Serqueira MMC. Aposentações por doença cardíaca em Portugal (2002-2008). Rev Port Cardiol. 2009;28(5):561-5.

19. Rywik TM, Kolodziej P, Targoński R, Fedyk-Lukasik M, Nowicka A, Zinka E, Zbyszyński B, Achremczyk P, Górski J, Muder A, Sadowski J, Leszek P, Kurjata P, Broda G, Korewicki J. Characteristics of the heart failure population in Poland - ZOPAN. Kardiol Polska. 2011;69(1):24-31.

20. Hung WW, Ross JS, Boockvar KS, Siu AL. Association of Chronic Diseases and Impairments with Disability in Older Adults: A Decade of Change? Med Care. 2012;50(6):501-7.

21. Gure TR, Blaum CS, Giordani B, Koelling TM, Galecki A, Pressler SJ, Hummel SL, Langa KM.The Prevalence of Cognitive Impairment in Older Adults with Heart Failure. J Am Geriatr Soc. 2012;60(9):1724-9. 